\title{
Evaluation of risk factors of anterior cruciate ligament (ACL) Injury: A prospective comparative analysis
}

\author{
Ashish Devgan ${ }^{1}$, Chanchal Barman ${ }^{2, *}$, Monish Malhotra ${ }^{3}$, Rajesh Rohilla ${ }^{4}$, Pradeep Yadav ${ }^{5}$ \\ ${ }^{1,4}$ Senior Professor, ${ }^{2,5}$ PG Resident, ${ }^{3}$ Senior Resident, Dept. of Orthopaedics, Pt. Bhagwat Dayal Sharma, PGIMS, Rohtak, \\ Haryana, India
}

*Corresponding Author: Chanchal Barman

Email: drchanchal002@gmail.com

\begin{abstract}
Introduction: Anterior Cruciate Ligament (ACL) is the most frequently injured knee ligament. Combination of valgus force with internal rotation is the most common mechanism involved in ACL injury. Both modifiable and non-modifiable risk factors are responsible for the ACL injury.

Purpose: To determine the risk factors causing ACL injury by comparing lower limb anatomical features, ACL size and body mass index (BMI) between ACL deficient and ACL intact knees.

Materials and Methods: Mechanical axis deviation from vertical axis, tibiofemoral angle, hip neck-shaft angle, posterior tibial slope, notch width index were compared using radiography. BMI and ultrasonographic (USG) diameter of ACL were also measured for comparison.

Results: Among the anatomical lower limb parameters mechanical axis deviation from vertical axis, hip neck shaft angle and tibiofemoral angle showed non-significant ( $\mathrm{p}$ value>0.05) differences while there were significant differences of femoral notch width index $(\mathrm{p}=0.014)$ and posterior tibial slope angle $(\mathrm{p}<0.001)$ between the two groups. ACL diameter (size) and BMI also showed significant differences $(\mathrm{p}<0.05)$. ACL deficient knees have higher BMI (mean 25.82 vs 23.33: $\mathrm{p}<0.001$ ) and lesser diameter (mean $7.31 \mathrm{~mm}$ vs $7.99 \mathrm{~mm}$; $\mathrm{p}<0.001$ ) compared to the ACL intact knees.

Conclusion: Higher posterior tibial slope and smaller notch width index are associated with higher risk of ACL injury. Individuals with lesser diameter of ACL or higher BMI have more chances of injury to the ligament.
\end{abstract}

Keywords: Anterior cruciate ligament (ACL), Risk factors, Body mass index, Posterior tibial slope (PTS), Notch width index (NWI).

\section{Introduction}

The risk factors causing Anterior Cruciate Ligament (ACL) injury may be modifiable or nonmodifiable. Modifiable risk factors determined previously include training regimens, skill level, neuromuscular biomechanics, proprioception, quadriceps and hamstring muscle strength ${ }^{1,2}$ which can be altered using non-invasive prophylactic interventions. ACL volume, posterior tibial slope (PTS), ${ }^{3,4}$ femoral intercondylar notch width ${ }^{2}$ are some of the studied non-modifiable risk factors which either cannot be altered or can be improved by surgical interventions only.

Translation of tibia over the femur anteriorly is primarily restrained by the ACL. A compressive load will create a greater anterior shear force which causes the tibia to translate anteriorly over the femur in a knee joint having increased posterior tibial slope. ${ }^{5}$ Therefore, a greater axial knee compressive force which may be due to greater body weight/BMI when combines with a higher posterior tibial slope will put the ACL at increased risk of tear. So, the relation between BMI and the knee anatomical parameters in the context of ACL injury risk is of particular interest which was addressed in this study. Chandrashekhar et al. ${ }^{6}$ determined that ACL size plays an important role in the risk of ACL injury, though it was a comparison made between two different genders. We evaluated the size of ACL near the tibial insertion site to compare it with ACL intact knees. In a study by Noyes FR et al. it was found that approximately $75 \%$ of ACL injuries were due to noncontact mechanism; which suggests earlier assessment may help to reduce injury prevalence and also in prevention of ACL injury. ${ }^{7}$

Thus, the aim of our study was to evaluate and identify significant modifiable or non-modifiable risk factors of ACL injury by comparing important anatomical features between the ACL intact and ACL deficient knee groups. Our study also aimed to relate the significance of BMI in presence of other risk factors in predicting ACL injury.

\section{Materials and Methods}

This study was done at a tertiary care center at Rohtak, Haryana in the department of Orthopedics. Lower limb anatomical features of 50 subjects having ACL deficient knees were compared with 50 normal subjects. Five anatomical parameters measured by radiography-mechanical axis deviation (MAD) from vertical axis, hip neck-shaft angle (NSA), tibiofemoral angle (TFA), posterior tibial slope (PTS) and notch width index (NWI) were chosen for comparison between the two groups. Both sides of ACL-deficient knees and the dominant side of ACL-intact knees were taken for measurements. We measured the diameter of ACL near its tibial attachment using ultrasonography (USG). Diameter of ACL in the non-injured side of the ACL deficient group was compared with the dominant 
side of the ACL intact group. Height and weight of each subject were taken for calculation of BMI and were compared between the two groups.

Inclusion Criteria: 50 Patients (46 males and 4 females) already diagnosed as ACL tear aged between 15 to 40 yrs. old were included as cases in group A.

50 normal subjects (46 males and 4 females) in same age group from the hospital staff were selected as controls in group B.

Exclusion Criteria: Patients having concomitant other ligamentous injury, generalized ligamentous laxity, patellar dislocations or injuries and those with prior lower limb deformities or injuries were excluded.

Exposure to the ionizing radiation during conventional radiography is minimal and has been proven to be safe in various studies done in past. ${ }^{8}$ So, the control population were well counselled regarding ethical concern before undergoing $\mathrm{x}$ rays. To reduce the inter-observer errors, measurements were taken by a single radiographer and repeated measurements were done to look for intra-observer variability.

Scannogram: Scannogram of the both the lower limbs exposing hip to ankle was taken. Mechanical axis deviation (MAD) from vertical axis, tibiofemoral angle (TFA), and hip neck-shaft angle (NSA) were measured from the scannogram. Centre of the femoral head and centre of the ankle joint is connected by a line to draw the mechanical axis. We measured the deviation of mechanical axis (MAD) from the vertical axis (Fig. 1a). The anatomical axis of femur is a line which bisects the intramedullary canal longitudinally into two equal halves or a line connecting the centre of femoral shaft to a point 10 centimetres proximal to the knee joint at the midpoint of medial and lateral cortex. ${ }^{9}$ Anatomical axis of tibia was drawn similarly. Angle formed between the anatomical axis of tibia and femur is called the tibiofemoral angle (TFA) (Fig. 1b). Hip neck-shaft angle (NSA) was drawn as the intersection of anatomical axis of femur and line drawn from centre of femoral head to mid-point of width of femoral neck (Fig. 1c).
Lateral View Radiograph: Standing lateral view was taken in $15^{\circ}-20^{\circ}$ knee flexion to avoid superimposition of the femoral condyles (Fig. 2a). A line along the anterior border of tibia and another line along the posterior slope of medial tibial plateau were drawn. The angle formed between the tibial slope and the perpendicular line drawn to the line along anterior tibial border was taken as PTS angle (Fig. 2b). For easier delineation medial tibial slope was preferred as compared to lateral tibial slope. ${ }^{10}$

Tunnel View of Knee: Subject was kneeled in $60^{\circ}$ flexion of the knee with the X-ray beam vertical to it from above (Fig. 3a). Notch width was measured along the line drawn parallel to the joint line at the level of popliteal groove, whereas femoral width was taken as the distance between the outermost borders of the femur along the same line (Fig. 3b). Notch width divided by femoral width was taken as notch width index (NWI). To represent notch stenosis as a risk factor of ACL injury we used the ratio of notch width and width of distal femur at the same level which minimises variabilities of magnification and differences in patients body stature or size. ${ }^{11}$

All the radiological parameters were measured using the Software RadiAnt DICOM Viewer 4.2.1(64bit). A mean value of 3 sets of measurements was taken to reduce intra-observer variability.

Diameter by USG: Diameter of the ACL nearest to its insertion over tibia was measured by ultrasound with the knee in $90^{\circ}$ flexion (Fig. 4a). All the ultrasonographic assessments were done by a single musculoskeletal radiologist. The linear transducer (7-9 $\mathrm{Hz}$ ) was placed over the skin just distal to patella maintaining its long axis parallel to that of the ligament by rotating the lower end of the probe medially by $30^{\circ}$ (Fig. 4b). ACL appeared as a thick linear hypoechoic band like structure extending from tibia towards femur (Fig. 4c).

BMI: We took the standing height and weight of the subjects and BMI was calculated (weight in $\mathrm{kg} / \mathrm{height}$ in $\mathrm{m}^{2}$ ).

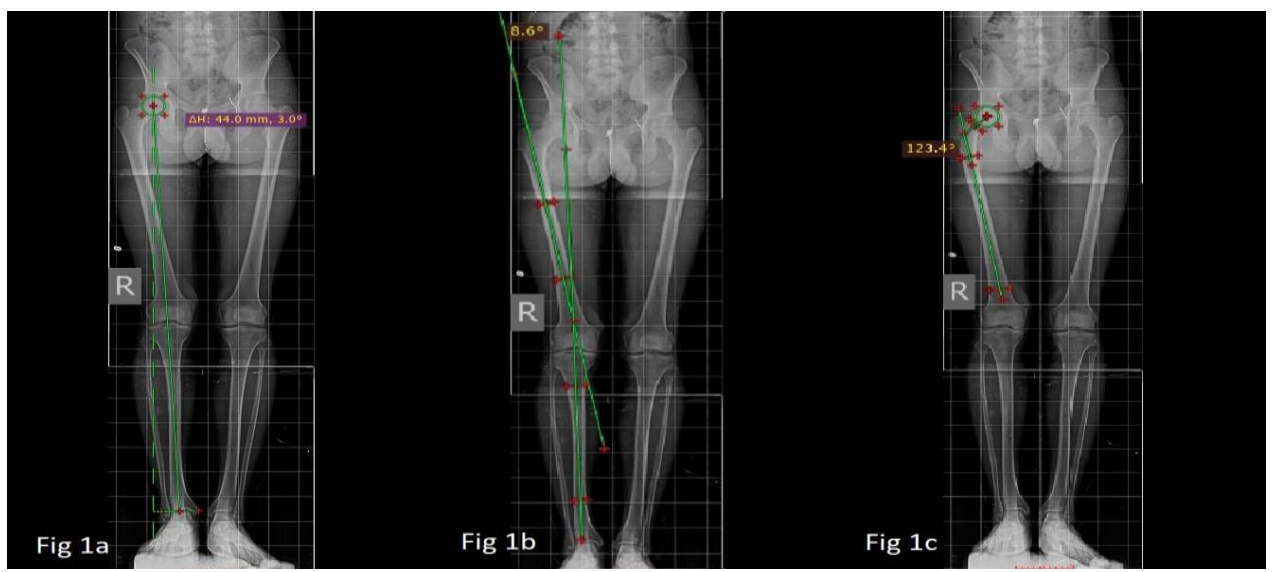

Fig. 1: Reference points taken for calculation of a): Mechanical axis deviation from vertical axis b): Tibiofemoral axis c): Hip neck shaft angle 


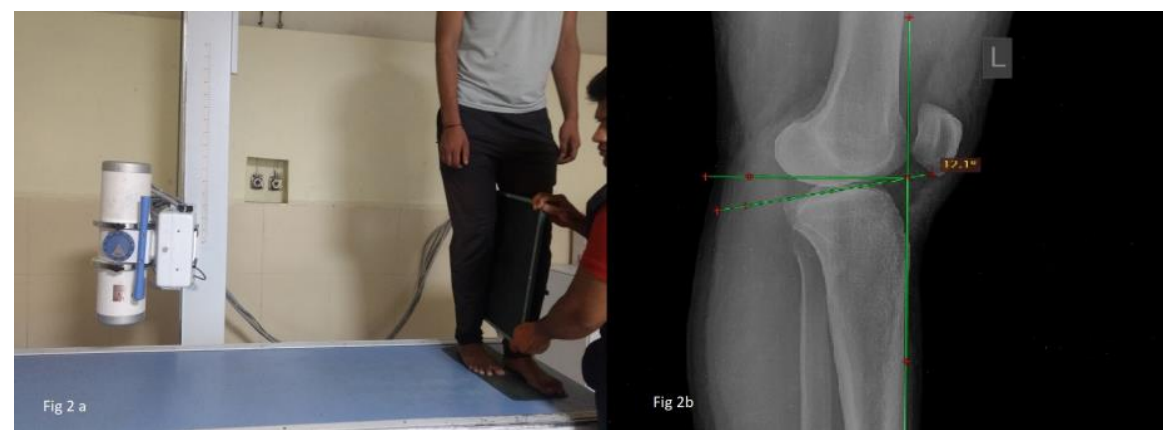

Fig. 2a): Position of the patient while taking lateral radiograph of the knee; b): Showing reference landmarks for measuring posterior tibial slope

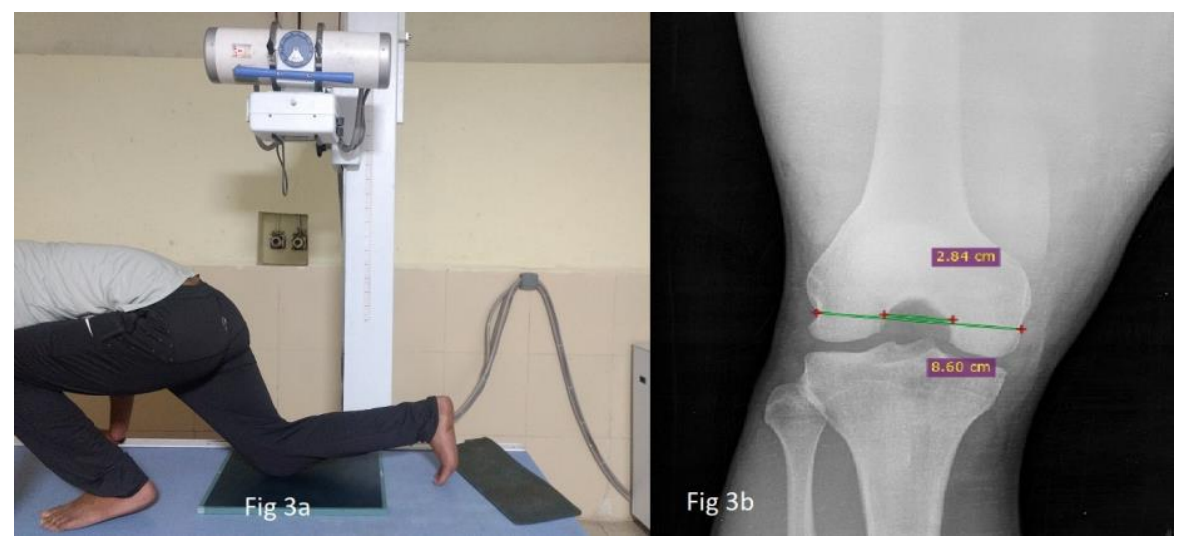

Fig. 3a): Position of the patient while taking tunnel view of knee joint; b): showing reference landmarks for calculation of femoral width and notch width

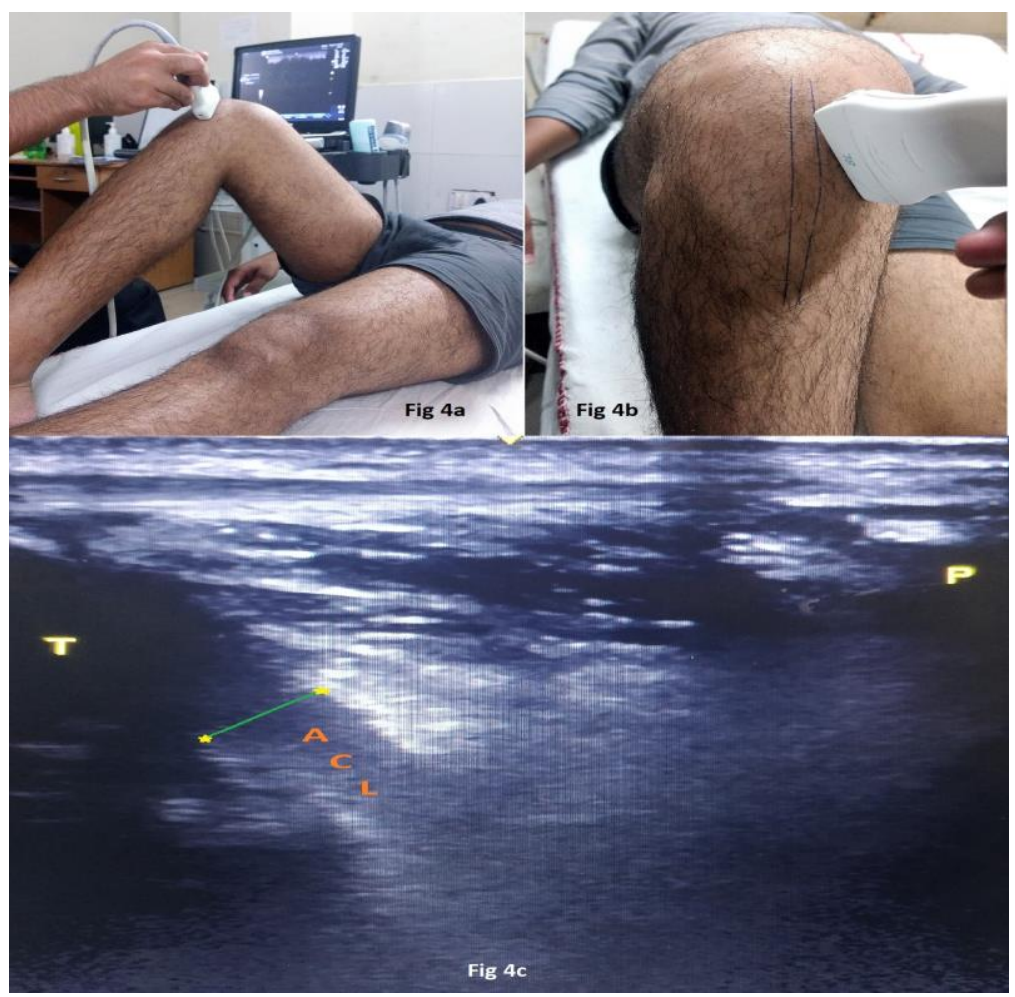

Fig. 4a): Flexing the knee to 90 degree while measuring diameter of ACL (size) near the tibial insertion; b): Shows rotation of the superior part of the linear probe externally by 30 degrees in order to make the probe parallel to ACL; c): Ultrasonographic assessment of width of ACL, appearing as a hypoechoic band at the tibial insertion; T-tibia, P-patella) 


\section{Statistical Analysis}

Students independent t-test was used to compare data between ACL deficient and intact group. A p value of $<0.05$ is considered as significant.

Univariate and multivariate logistic regression were used to assess correlation among body mass index (BMI), posterior tibial slope (PTS) and risk of ACL injury. Variables found significant in univariate analysis were employed in multivariate logistic regression analysis to see their contribution towards variance of the dependent variables. Data were analysed using SPSS (Statistical Packages for Social Sciences) for Windows; version 17.0 (Chicago: SPSS Inc).

\section{Results}

We found no significant difference between the injured and non-injured sides of ACL deficient group (Table 1). Significant differences ( $p$ value $<0.05$ ) in posterior tibial slope (PTS) and notch width index (NWI) were found while comparing the ACL deficient knees (group A) with ACL intact knees (group B). The
PTS (p value $<0.001$ ) was significantly larger and NWI ( $p$ value 0.014 ) was significantly smaller in ACLdeficient knees (Table 2). The ACL deficient subjects had greater BMI values than the normal subjects with significant difference (mean value of BMI 25.82 vs 23.33; $p$ value $<0.001$ ) (Table 3). Results of univariate logistic regression analysis revealed that PTS $(\mathrm{p}=<0.001)$ and BMI $(\mathrm{p}=<0.001)$ both interplay an important role causing ACL injury as both the parameters showed significant difference between the two groups. (Table 4). Predicted increase in BMI by one unit increases the injury risk by $25 \%$ when other factors such as PTS is kept constant whereas one degree increase in PTS increases the injury risk by $38 \%$ when BMI is kept constant (Table 5).

Evaluation of diameter of ACL near tibial insertion showed significant difference between ACL deficient and intact knees (mean $7.31 \mathrm{~mm}$ vs $7.99 \mathrm{~mm}$; p value $<0.001$ ). ACL injured subjects were found to have comparatively thin (less diameter) ligaments (Table 6).

Table 1: Comparison of lower limb anatomical features between Injured and Non-injured sides of the ACL deficient knee group (group-A):

\begin{tabular}{|l|c|c|c|c|}
\hline Anatomical parameters & Side & Mean & Std. Deviation & P-value \\
\hline MAD & Injured & 3.34 & 0.84 & 0.478 \\
& Non-injured & 3.21 & 0.87 & \\
TFA & Injured & 5.28 & 2.32 & 0.336 \\
& Non-injured & 5.73 & 2.32 & \\
PTS & Injured & 13.15 & 2.97 & 0.735 \\
& Non-injured & 13.36 & 2.99 & \\
NWI & Injured & 0.3077 & 0.0265 & 0.083 \\
& Non-injured & 0.3185 & 0.0344 & \\
NSA & Injured & 128.61 & 4.90 & 0.161 \\
& Non-injured & 127.26 & 4.68 & \\
\hline
\end{tabular}

MAD=Mechanical axis deviation; TFA=Tibiofemoral angle; PTS= Posterior tibial slope; NWI=Notch width index; NSA $=$ Neck-shaft angle

Table 2: Comparison of lower limb anatomical features between ACL deficient (group A) vs ACL intact knees (group B):

\begin{tabular}{|l|c|c|c|c|}
\hline Anatomical parameters & Group & Mean & $\begin{array}{c}\text { Std. } \\
\text { Deviation }\end{array}$ & P-value \\
\hline MAD & ACL deficient & 3.34 & 0.84 & 0.886 \\
& ACL intact & 3.36 & 0.83 & \\
TFA & ACL deficient & 5.28 & 2.32 & 0.324 \\
& ACL intact & 6.32 & 7.02 & \\
PTS & ACL deficient & 13.15 & 2.97 & $<0.001$ \\
& ACL intact & 10.87 & 2.27 & \\
NWI & ACL deficient & 0.3077 & 0.0265 & 0.014 \\
& ACL intact & 0.3210 & 0.0268 & \\
NSA & ACL deficient & 128.61 & 4.90 & 0.272 \\
& ACL intact & 129.67 & 4.66 & \\
\hline
\end{tabular}

MAD=Mechanical axis deviation; TFA=Tibiofemoral angle; PTS= Posterior tibial slope; NWI=Notch width index; NSA $=$ Neck-shaft angle 
Table 3: Comparison of BMI between ACL deficient vs ACL intact knees (group A vs B):

\begin{tabular}{|l|c|c|c|c|}
\hline Variables & Group & Mean & Std. Deviation & P-value \\
\hline Age & ACL deficient & 25.16 & 5.05 & 0.646 \\
& ACL intact & 24.70 & 4.93 & \\
BMI & ACL deficient & 25.82 & 3.62 & $<0.001$ \\
& ACL intact & 23.33 & 3.05 & \\
\hline
\end{tabular}

BMI=Body mass index

Table 4: Univariate logistic regression models of variables and their odds' ratio with $95 \%$ confidence interval:

\begin{tabular}{|l|c|c|c|}
\hline Variable & Mean \pm SD & P-value & Odd's ratio (95\% CI) \\
\hline BMI & $25.82 \pm 3.62$, cases & & \\
& $23.33 \pm 3.05$, control & $<0.001$ & $1.254(1.097-1.433)$ \\
PTS & $13.15 \pm 2.97$, cases & $<0.001$ & $1.391(1.168-1.655)$ \\
& $10.87 \pm 2.27$, controls & $<.001$ & \\
\hline
\end{tabular}

PTS $=$ Posterior tibial slope; BMI=Body mass index

Table 5: Predicted increase in ACL injury risk (Multivariate logistic regression analysis):

\begin{tabular}{|l|c|c|c|}
\hline BMI, Kg/m & \% Increase & PTS, degree & \% Increase \\
\hline+1 & 25 & +1 & 38 \\
+2 & 50 & +2 & 76 \\
+3 & 75 & +3 & 114 \\
\hline
\end{tabular}

Table 6: Comparison of diameter of ACL between ACL deficient vs ACL intact knees (group A vs B) using ultrasonography:

\begin{tabular}{|l|c|c|c|c|}
\hline & Group & Mean (in mm) & Std deviation & p-value \\
\cline { 2 - 5 } Diameter of ACL near & ACL deficient & 7.31 & 0.681 & \\
tibial insertion & ACL intact & 7.99 & 0.621 & $<0.001$ \\
\hline
\end{tabular}

\section{Discussion}

ACL injury has much higher incidence rate than other ligamentous structure around the knee joint. ${ }^{12}$ Understanding the mechanism and predisposing factors of non-contact ACL injury are of greater importance for patients and clinicians both as it helps to design neuromuscular training programs specially for athletes. ${ }^{13}$

In this study, we assumed that before injury both the lower limbs were comparable in terms of anatomical features or lower limb anatomical alignments. Comparison of injured and non-injured sides of the ACL deficient knees (table 1) showed nonsignificant differences $(p>0.05)$, which explains injury to the ligament itself is unable to cause any alteration in the anatomical features or alignments of the lower limbs. Hence, for comparison, choice of any of the two knees of the control subjects (we have chosen dominant one) in our study is justified.

Varus alignment may not cause increased load on the ACL due to smaller Q angle. ${ }^{14}$ Alignment of the knee was observed to be in more varus in Chinese and Japanese subjects as compared to the Caucasians, though the risk of ACL injury among these populations was not significantly diferrent. ${ }^{15,16}$ We measured anatomical TFA for comparison but it showed no significant difference between the two groups. However, it is stated that measurement of a dynamic tibiofemoral angle had greater importance than static knee alignment while considering ACL injuries by noncontact mechanisms. ${ }^{10}$ Similarly, we noticed no significant difference in mechanical axis deviation (MAD) form vertical axis and hip neck-shaft angles (NSA) among both the groups. Static Q-angle neither correlates with knee valgus nor predicts patterns of ACL injury risk ${ }^{17,18}$, and hence was not evaluated in our study.

The risk of ACL injury increases in presence of a narrower intercondylar notch. ${ }^{19}$ We noticed comparatively lower values of notch width index (NWI) in ACL injured subjects. This was significantly smaller ( $p$ value $<0.05$ ) as compared to ACL intact knees. Different mechanisms have been suggested how a knee with smaller notch width index predisposes the ligament to injury. A narrower notch causes repeated impingement of the ligament, particularly in extension of the knee. ${ }^{11}$ Some believe notch width correlates with the size of the ACL and thereby the strength of the ligament. ${ }^{20}$ Those with a tighter notch probably have the ligament in more coronal plane, thus making them more prone to loads due to its vertical alignment. ${ }^{21}$

Posterior tibial slope (PTS) correlates biomechanically with ACL injury risk and it has been described that higher tibial slope causes increased risk of ACL injury. ${ }^{22,23}$ Dejour and Bonnin et al. ${ }^{5}$ used lateral radiographs to demonstrate more anterior translation of the tibia with higher posterior tibial slope angles by comparing the ACL-deficient subjects with 
normal population. They noticed, increasing PTS by 10 degrees increases $6 \mathrm{~mm}$ anterior translation of the tibia over femur and three times increase in ACL load. McLean et al. ${ }^{24}$ also found the same and reported increased anterior tibial acceleration with increase in PTS. Shelburne et al. ${ }^{25}$ and Shao et al. ${ }^{26}$ used computer modelling and electromyography and they demonstrated an increased ACL strain with an increase in PTS during both standing and moving postures.

Only a few numbers of studies were able to demonstrate how variation in the size of ACL predisposes the ligament to get injured. ${ }^{6,27}$ Chandrasekhar et al. compared $A C L$ volume between males and females and they noticed a significant gender differences in ACL volume among the two groups. It was the first study to demonstrate size of the ligament can influence the chance of injury. ${ }^{6}$ Also ACL was found to be thinner in females and thus predisposing them to such injuries. ${ }^{27,28}$ With this thought, a thinner ACL was considered to be a risk factor causing injury. It had been evaluated in some studies that the mean fibril diameter is uniform among both the $\operatorname{sexes}^{29}$ and we believe that it is the number of fibrils within the ACL which determines its thickness is more important. In our study, though majority of the patients were males it was a comparison between ACL deficient versus intact knees and here we observed that size of the ligament was significantly lesser in ACL deficient knees. In other words, an ACL having less diameter predisposes the individual more prone to injury.

Measuring the diameter of the already injured ACL is erroneous, we took diameter of the uninjured ACL from the other normal limb to represent the diameter of the injured ACL. In a study by Jamison et al. bilateral knees ACL size were compared using MRI in healthy subjects and they noticed no significant difference in size of the two ACLs of a same subject. ${ }^{30}$ Therefore, the contralateral ACL can be taken as a substitute for the injured ACL for measuring size or diameter and we did the same. Our study results demonstrate that ACL injured subjects have smaller ACL diameter in their contralateral normal knees, clarifying a thinner ACL is comparatively weak and it is more predisposed to injury.

BMI and knee morphometry have an obvious association in the causation of ACL injury. ${ }^{31}$ BMI, being a modifiable factor, prevention strategies can be planned for ACL injuries especially in population who are at risk (like sportspersons or athletes) of such injuries. Study results suggest that an individual with higher BMI has more chance of ACL injury when associated with increased posterior tibial slope and hence it can be stated that BMI influences the correlation between PTS and ACL injury risk. PTS is an anatomical bony morphology which is nonmodifiable. Whereas BMI is calculated from measurements of weight and height of the individual, of which weight can be modified accordingly. This is of great importance for those athletes who are at risk of having non-contact ACL injuries. They are trained to increase or decrease weight, and thereby to modify $\mathrm{BMI}$, to improve their athletic performances. Injury prevention strategies can include those individuals who are having higher PTS and at the same time greater BMI values.

In their study Bojicic KM et al. (2017) showed that an increased fat body mass in a subject with already having a higher PTS causes more chance of injury; while an increase in lean body mass may not exacerbate injury risk, rather it might even decrease the chance of injury. ${ }^{31}$ Most of the athletes concentrate on building lean body mass to gain strength and improve on their fitness to prevent injuries during activities. Increased body weight due to gain of muscle mass should be in a balance with increased injury risk especially when associated with a higher PTS. Identification of steep PTS in patients who are already at increased risk can be of paramount importance in preventing injury to ACL by identifying such individuals and giving them targeted neuromuscular therapy. But a gain in the fat body mass only is undesirable and it should be further evaluated in future research. However, in individuals already having increased slope, a higher BMI is an important risk factor to them as it can be subjected to modification accordingly. Our study predicts how an increase in BMI alone will contribute or increase in the probability of injury risk, keeping PTS as constant and vice versa.

It is important to the athletic population to have proper assessment for risk factors through screening. Neuromuscular exercises to decrease stress on the ligament are described to reduce the chances of injury. ${ }^{1}$ Exercises of specific muscle groups working in synergy with the ACL minimises strain over the ligament and thus may help to curb the risk of injury. ${ }^{32}$

MRI appears to be more appropriate for measurement of ACL diameter; however, for a large population MRI as a screening tool is not ideal being too expensive. Moreover, for a healthy athlete without any obvious symptoms doing MRI is still a matter of debate and is not justified. Hence ultrasonography can be the right choice for screening purpose, because it is more economical, easily available.

\section{Limitations of the Study}

1. 'Diameter' was chosen to represent the 'size' and we measured the diameter of only near the tibial insertion of the ligament by ultrasonography, which were the major limitations of this study. Shearing force on the ligament divided by its crosssectional area correctly determines the importance 'size' as a risk factor. But, measuring crosssectional area using ultrasound is not possible as it cannot clearly distinguish ACL from adjacent soft tissues. Rather 'volume' could be a good alternative to represent size of ACL; however, it is 
not possible to measure ACL volume by ultrasound. ${ }^{33}$

2. The injury mechanism was determined by eliciting history from the injured patients. Types or levels of physical activity may influence the mechanical properties more importantly the tensile strength of the ligament due to recurrent loading. So, there is a possibility that subjects who were sportspersons by profession might have stronger ACL than those who were not associated with athletic activities. This discrepancy was not considered in our study. Level of activities before injury between the two groups were matched only by assumption. Subjects from the control group have a possibility that they may suffer ACL injury in future. Age was also matched among the two groups to decrease the disparity.

3. Posterior tibial slope is three-dimensional entity which should be measured at the center of the articular regions for medial and lateral compartments of the plateau. MRI is preferred as there is superimposition of the plateau on radiographs. However, since we did not obtain MRI for control group in our study, we calculated as per described PTS measurement in lateral view of knee joint.

\section{Future Prospects}

1. Greater weight (or higher BMI) whether may it be because of increased lean body mass or increased fat body mass, or an increase in both, differently modifies the risk of ligamentous injury when combined with a steeper PTS is yet to be determined in further studies.

2. Questions like which type of weight (whether fat or lean body mass) and how much reduction or gain in weight is detrimental to an athlete in context of ACL injury risk, are still a matter of debate and it can be the target of future studies.

\section{Conclusion}

ACL injury is associated with multiple risk factors. Individuals having greater PTS and narrower NWI are at increased risk of ACL injury. ACL injured subjects are having comparatively thin (lesser diameter) ACL. Higher values of BMI in ACL injured subjects suggest increased BMI also predisposes to injury of the ligament. The risk of ACL injury has an obvious positive relationship with increased PTS combined with increased BMI and vice versa.

\section{Acknowledgement}

The authors thank the statistician; and the radiographers for their assistance.

\section{Conflict of interest: None.}

Funding: There was no funding source for this study.

\section{References}

1. Hewett TE, Lindenfeld TN, Riccobene JV, Noyes FR. The effect of neuromuscular training on the incidence of knee injury in female athletes: A prospective study. Am J Sports Med. 1999;27:699-706.

2. Anderson AF, Dome DC, Gautam S, Awh MH, Rennirt GW. Correlation of anthropometric measurements, strength, anterior cruciate ligament size, and intercondylar notch characteristics to sex differences in anterior cruciate ligament tear rates. Am J Sports Med. 2001;29:58-66.

3. Hohmann E, Bryant A, Reaburn P, Tetsworth K. Is there a correlation between posterior tibial slope and noncontact anterior cruciate ligament injuries? Knee Surg, Sports Traumatol, Arthrosc. 2011;19:109-114.

4. Hudek R, Fuchs B, Regenfelder F, Koch PP. Is noncontact ACL injury associated with the posterior tibial and meniscal slope? Clin Orthop Relat Res. 2011; 469:2377-84.

5. Dejour H, Bonnin M. Tibial translation after anterior cruciate ligament rupture. Two radiological tests compared. J Bone Joint Surg Br. 1994;76:745-759.

6. Chandrashekar N, Slauterbeck J, Hashemi J. Sex-based differences in the anthropometric characteristics of the anterior cruciate ligament and its relation to intercondylar notch geometry: a cadaveric study. Am J Sports Med. 2005;33:1492-498.

7. Noyes FR, Mooar PA, Matthews DS, Butler DL. The symptomatic anterior cruciate-deficient knee. Part I: the long-term functional disability in athletically active individuals. JBJS. 1983;65:154-162.

8. Amis Jr ES, Butler PF, Applegate KE et al. American College of Radiology white paper on radiation dose in medicine. J Am Coll Radiol. 2007;4:272-84.

9. Luo CF. Reference axes for reconstruction of the knee. The Knee. 2004;11:251-257.

10. Chung SC, Chan WL, Wong SH. Lower limb alignment in anterior cruciate ligament-deficient versus-intact knees. J Orthop Surg. 2011;19:303-308.

11. Souryal TO, Moore HA, Evans JP. Bilaterality in anterior cruciate ligament injuries: associated intercondylar notch stenosis. Am J Sports Med. 1988;16:449-454.

12. Johnson RJ. The anterior cruciate ligament problem. Clin Orthop Relat Res. 1983;172:14-18.

13. Shimokochi Y, Shultz SJ. Mechanisms of noncontact anterior cruciate ligament injury. J Athl Train. 2008;43:396-408.

14. Jan van de Pol G, Arnold MP, Verdonschot N, van Kampen A. Varus alignment leads to increased forces in the anterior cruciate ligament. Am J Sports Med. 2009;37:481-487.

15. Hovinga KR, Lerner AL. Anatomic variations between Japanese and Caucasian populations in the healthy young adult knee joint. J Orthop Res. 2009;27:1191-1196.

16. Tang WM, Zhu YH, Chiu KY. Axial alignment of the lower extremity in Chinese adults. J Bone Joint Surg Am. 2000;82:1603-1608.

17. Gray J, Taunton JE, McKenzie DC, Clement DB, McConkey JP, Davidson RG. A survey of injuries to the anterior cruciate ligament of the knee in female basketball players. Int J Sports Med. 1985;6:314-316.

18. Myer GD, Ford KR, Hewett TE. The effects of gender on quadriceps muscle activation strategies during a maneuver that mimics a high ACL injury risk position. $J$ Electromyogr Kinesiol. 2005;15:181-189.

19. Palmer I. On the injuries to the ligaments of the knee joint. A clinical study. Acta Chir Scand Supple. 1938;53:1-28. 
20. Stijak L, Herzog RF, Schai P. Is there an influence of the tibial slope of the lateral condyle on the ACL lesion? Knee surg, sports traumatol, arthrosc. 2008;16:112-117.

21. Simon RA, Everhart JS, Nagaraja HN, Chaudhari AM. A case-control study of anterior cruciate ligament volume, tibial plateau slopes and intercondylar notch dimensions in ACL-injured knees. J Biomech. 2010;43:1702-1707.

22. Beynnon BD, Hall JS, Sturnick DR. Increased slope of the lateral tibial plateau subchondral bone is associated with greater risk of noncontact ACL injury in females but not in males: a prospective cohort study with a nested, matched case-control analysis. Am J Sports Med. 2014;42:1039-1048.

23. Hashemi J, Chandrashekar N, Mansouri H. Shallow medial tibial plateau and steep medial and lateral tibial slopes: new risk factors for anterior cruciate ligament injuries. Am J Sports Med. 2010;38:54-62.

24. McLean SG, Oh YK, Palmer ML. The relationship between anterior tibial acceleration, tibial slope, and ACL strain during a simulated jump landing task. J Bone Joint Surg Am. 2011;93:1310-1317.

25. Shelburne KB, Kim HJ, Sterett WI, Pandy MG. Effect of posterior tibial slope on knee biomechanics during functional activity. J Orthop Res. 2011;29:223-231.

26. Shao Q, MacLeod TD, Manal K, Buchanan TS. Estimation of ligament loading and anterior tibial translation in healthy and ACL-deficient knees during gait and the influence of increasing tibial slope using EMG-driven approach. Annals of Biomedical Engineering. 2011;39:110-121.

27. Chandrashekar N, Mansouri H, Slauterbeck J, Hashemi J. Sex-based differences in the tensile properties of the human anterior cruciate ligament. J Biomech. 2006; 39:2943-2950.

28. Dienst M, Schneider G, Altmeyer K. Correlation of intercondylar notch cross sections to the ACL size: a high-resolution MR tomographic in vivo analysis. Arch
Orthop Trauma Surg. 2007;127:253-260.

29. Hashemi J, Chandrashekar N, Mansouri H, Slauterbeck JR, Hardy DM. The human anterior cruciate ligament: sex differences in ultrastructure and correlation with biomechanical properties. J Orthop Res. 2008;26:945950.

30. Jamison ST, Flanigan DC, Nagaraja HN, Chaudhari AM. Side-to-side differences in anterior cruciate ligament volume in healthy control subjects. J Biomech 2010; 43:576-578.

31. Bojicic KM, Beaulieu ML, Imaizumi Krieger DY, Ashton-Miller JA, Wojtys EM. Association between lateral posterior tibial slope, body mass index, and ACL injury risk. Orthop J Sport Med. 2017;5:1-7.

32. Chaudhari AM, Zelman EA, Flanigan DC, Kaeding CC, Nagaraja HN. Anterior Cruciate Ligament-Injured Subjects Have Smaller Anterior Cruciate Ligaments than Matched Controls: A Magnetic Resonance Imaging Study. Am J Sports Med. 2009;37:1282-1287.

33. Mahajan PS, Chandra P, Negi VC, Jayaram AP, Hussein SA. Smaller anterior cruciate ligament diameter is a predictor of subjects prone to ligament injuries: an ultrasound study. Bio Med Res Int. 2015;2015.

How to cite this article: Devgan A, Barman C, Malhotra M, Rohilla R, Yadav P. Evaluation of risk factors of anterior cruciate ligament (ACL) Injury: A prospective comparative analysis. Indian $\mathrm{J}$ Orthop Surg. 2018;4(4):354-361. 